\title{
CAB models for water: new models for the interaction of thermal neutrons in water
}

\author{
J.I. Márquez Damián*† \\ Departamento Física de Neutrones and Instituto Balseiro, Centro Atómico Bariloche, Bariloche, \\ Argentina \\ E-mail: marquezj@cab.cnea.gov.ar
}

\section{J.R. Granada}

Departamento Física de Neutrones and Instituto Balseiro, Centro Atómico Bariloche, Bariloche, Argentina

\section{D.C. Malaspina}

Computation, Modeling \& Bioinformatics Center, Northwestern University, Evanston, IL, USA

\begin{abstract}
During the past few years we have been working at the Department of Neutron Physics at Centro Atómico Bariloche on an update of the evaluation of the thermal neutron scattering law for light and heavy water. We based our approach on combining the best available experimental data with molecular dynamics simulations. With this methodology we produced a set of new thermal neutron scattering files in ENDF-6 format, that result an improvement over existing evaluations.
\end{abstract}

In this paper we present the models, a brief summary of the validation and some applications to nuclear reactor calculations.

X Latin American Symposium on Nuclear Physics and Applications (X LASNPA),

1-6 December 2013

Montevideo, Uruguay

${ }^{*}$ Speaker.

${ }^{\dagger}$ Also at CONICET. 


\section{Introduction}

The Neutron Physics Department at Centro Atómico Bariloche has a long history working on the development of thermal scattering libraries [1-3]. During the past five years we applied this experience to the improvement of thermal scattering libraries for light and heavy water. As a result we produced a new set of models, the $C A B$ models for water.

This work has been published in part in Ref. [4] (calculation of frequency spectra from molecular dynamics simulations) and in Ref. [5] (models and validation). The reader is referred to these papers for a more in-depth analysis.

\section{Existing models}

The double differential scattering cross section gives the probability of interactions that change the energy and direction of thermal neutrons from $E$ and $\Omega$, to $E^{\prime}$ and $\Omega^{\prime}$. This cross section is usually expressed as the product of a bound scattering cross section $\left(\sigma_{b}\right)$, which is the probability of interaction of neutrons with one stationary nucleus, and a function called scattering law $(S(\alpha, \beta))$ :

$$
\frac{\mathrm{d}^{2} \sigma}{\mathrm{d} E \mathrm{~d} \hat{\Omega}}=\frac{\sigma_{b}}{4 \pi k T} \sqrt{\frac{E^{\prime}}{E}} S(\alpha, \beta)
$$

The scattering law is a property of the material, and it is a function of the non-dimensional change in momentum $(\alpha)$ and energy $(\beta)$ :

$$
\begin{gathered}
\alpha=\frac{E+E^{\prime}-2 \sqrt{E^{\prime} E} \cos \theta}{A k_{B} T} \\
\beta=\frac{E^{\prime}-E}{k_{B} T}
\end{gathered}
$$

where $A$ is the ratio of the mass of the scatterer to the mass of the neutron, $T$ is the temperature of the material and $k_{B}$ is the Boltzmann constant.

In the evaluated nuclear data libraries, the thermal scattering law is distributed as part of the thermal scattering sublibrary, using the File 7 section of the ENDF-6 format [6]. Despite the importance of thermal scattering in water for nuclear systems, the scattering law files currently available in the different evaluated nuclear data libraries are still being calculated from two essentially different models: one proposed by General Atomics in the 1970's [7], and the other initially proposed by IKE Stuttgart in the 1980's [8].

Currently four of the major evaluated nuclear data libraries contain a thermal scattering sublibrary: JENDL [9], JEFF [10], ENDF/B [11] and ROSFOND [12]. The latest version of the JENDL library, JENDL-4.0, was released in 2010 and included the scattering law files for water extracted from the ENDF/B-VI.8 library, which were evaluated using a version General Atomics model to run using the LEAPR module of NJOY [13]. The new version of the European nuclear data library, JEFF-3.2, was released in March 2014 and includes thermal scattering data evaluated by Keinert and Mattes in 2004 using the IKE Stuttgart model [14]. ENDF/B-VII.1 includes thermal scattering data for water evaluated by Macfarlane using a modified version of the IKE Stuttgart model. The 
latest version of the Russian library, ROSFOND-2010, includes thermal scattering files for water adopted from ENDF/B-VII.

These models provide a good description of the scattering of thermal neutrons in water, but in the last few years there has been some concern raised on the accuracy of these evaluations, specially for heavy water $[15,16]$.

\section{Proposed models: $C A B$ models for water}

To improve on the existing situation we propose a new set of models, the $C A B$ models for water, which combine results from molecular dynamics simulations with experimental data.

The frequency spectra on which these models are based was computed from molecular dynamics results. Using the molecular dynamics program GROMACS [17] and the flexible water potential TIP4P/2005-flexible [18] we simulate systems of $512 \mathrm{H}_{2} \mathrm{O}$ and $\mathrm{D}_{2} \mathrm{O}$ molecules at a given temperature and pressure, and calculate the trajectories of the atoms. From this, we compute the velocity autocorrelation functions:

$$
\operatorname{VACF}_{\alpha}(\tau)=\left\langle v_{\alpha}(t) \cdot v_{\alpha}(t+\tau)\right\rangle
$$

where $\langle\cdot\rangle$ is the ensemble average over each nuclide $\left({ }^{1} \mathrm{H},{ }^{2} \mathrm{H}\right.$ and $\left.{ }^{16} \mathrm{O}\right)$.

Using these results, the frequency spectra is computed as the cosine Fourier transform of the VACF:

$$
\rho(\varepsilon)=\frac{M}{3 \pi k T} \frac{1}{2 \pi} \int_{0}^{\infty} \operatorname{VACF}(\tau) \cos (\omega \tau) d \tau
$$

where $\varepsilon=E^{\prime}-E=\hbar \omega$ is the excitation energy.

The model for light water is a model for $\mathrm{H}$ in $\mathrm{H}_{2} \mathrm{O}$ and uses the calculated spectra for hydrogen in light water, combined with the Egelstaff-Schofield diffusion [19] model to compute the scattering law in the incoherent approximation. The diffusion mass used in the model was obtained from measurements by Novikov [20].

The model for heavy water is a model for $\mathrm{D}$ and $\mathrm{O}$ in $\mathrm{D}_{2} \mathrm{O}$ that uses uses the calculated frequency spectra for deuterium and oxygen bound in heavy water. The diffusion component is computed using the Egelstaff-Schofield diffusion model, using a diffusion mass that was estimated from the results for light water:

$$
m_{\text {diff }}^{\mathrm{D}_{2} \mathrm{O}}=\frac{m_{\mathrm{D}_{2} \mathrm{O}}}{m_{\mathrm{H}_{2} \mathrm{O}}} m_{\text {diff }}^{\mathrm{H}_{2} \mathrm{O}}
$$

The coherent component of the scattering cross section is calculated applying the Sköld approximation [21], with partial structure factors obtained by Soper [22].

\section{Results and applications}

The models presented here were validated using experimental data from double differential, quasi-elastic, differential and total neutron cross section measurements. The results of this validation can be found in Ref. [5]. 


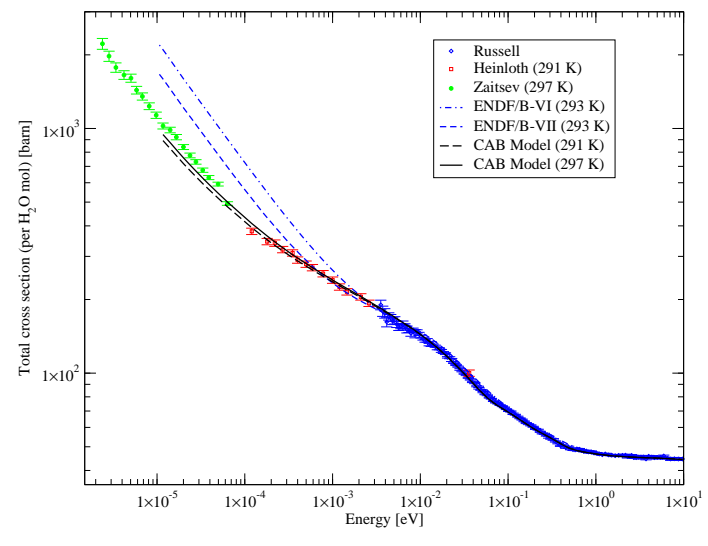

(a) Total neutron cross section for $\mathrm{H}_{2} \mathrm{O}$.

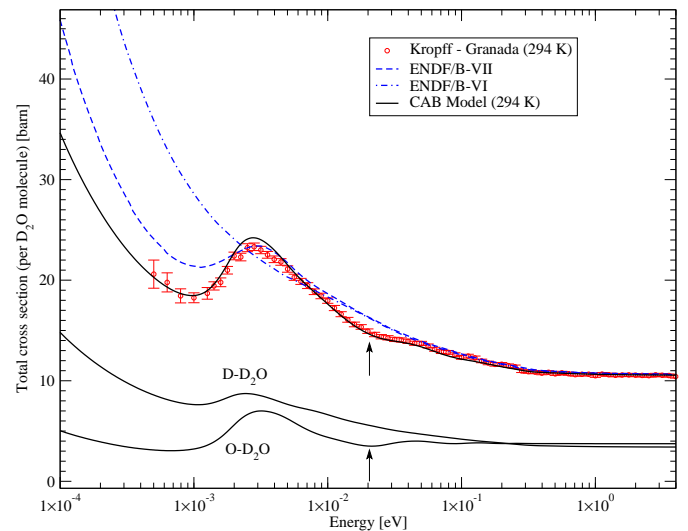

(b) Total neutron cross section for $\mathrm{D}_{2} \mathrm{O}$.

Figure 1: Total neutron cross section for light water (left) and heavy water (right) at room temperature.

As an example we show here results for the calculation of the total neutron cross section for light water (Fig. 1(a)) compared with measurements by Russell (EXFOR\# 11162003), Heinloth (EXFOR\# 21341002) and Zaitsev [23] around room temperature (Fig. 1(a)). Our model reproduces better the measured values of the total cross section in the cold neutron $(E<1 \mathrm{meV})$ range, which are overpredicted by the General Atomics and IKE models by 60 to $100 \%$.

For heavy water (Fig. 1(b)), our model reduces the discrepancies found between the existing models and the measurements by Kropff (EXFOR\# 30283001). Our model reproduces well the peak found at $\sim 3 \mathrm{meV}$, but also the features observed around $20 \mathrm{meV}$, which are caused by the coherent effects in the oxygen cross section (marked with arrows), and not included in the existing libraries.

To test these cross section libraries we ran a group of selected benchmarks from the International Criticality Safety Benchmark Evaluation Project (ICSBEP) [24]. The ICSBEP is a NEAOECD project to compile, analyze and document criticality experiments to be used as benchmarks. The results of this process are more than 400 benchmarks compiled in the International Handbook of Evaluated Criticality Safety Benchmark Experiments (ICSBEP Handbook), which is edited and distributed every year as a DVD. These benchmarks include a description of the relevant physical parameters and their uncertainties, and an analysis of the effect of these uncertainties in the multiplication factor of the system. For each configuration the expected multiplication factor is given ( $k_{\text {bench }}$ ), along with an uncertainty value that includes both the experimental uncertainties and the effect of the uncertainties in the different parameters of the system ( $\left.\delta k_{\text {bench }} \geq \delta k_{\text {exp }}\right)$.

To study the effect of the thermal scattering library (TSL) for light water we selected a group of light water moderated thermal systems with small benchmark uncertainty ( $\delta k_{\text {bench }} \lesssim 100 \mathrm{pcm}$ ). The benchmark comprised 11 uranium dioxide fueled systems (from benchmarks LEU-COMPTHERM-054, -058 and -079), one uranium metal system (LEU-MET-THERM-007), 26 uranium nitrate solution systems (LEU-SOL-THERM-004, -007, -011, -019, -021, -023, -024 and -025), 4 systems with uranium dioxide fuel rods and uranium nitrate solutions (LEU-MISC-THERM-001, $-002,-003$ and -005), 40 systems with MOX fuel rods in plutonium nitrate solutions (MIX-MISCTHERM-002 and -003) and 4 systems with plutonium nitrate solutions (PU-SOL-THERM-012). 
The systems were calculated using ENDF/B-VII cross sections and the neutron transport code MCNP5-1.60 [25]. The results show (Fig. 2) an overall similar agreement when the thermal scattering library from ENDF/B-VII is replaced by our library, with differences in the calculations below $100 \mathrm{pcm}$. In 49 of the 86 systems (57\%) the computed multiplication factor was closer to the benchmark value when the new library was used.

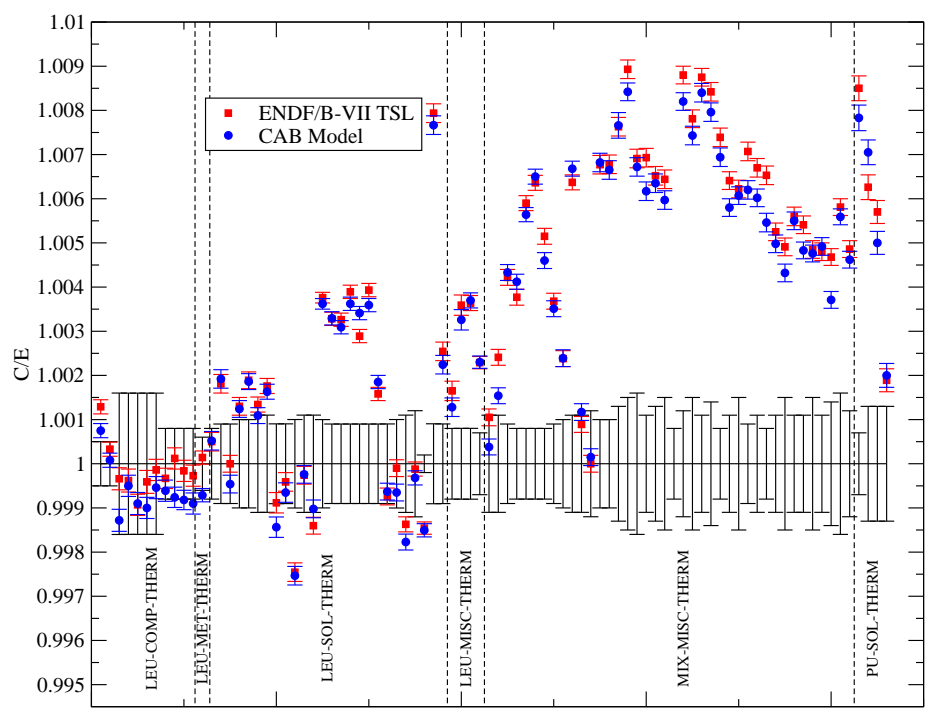

Figure 2: Calculated over experimental (benchmark) ratio for the multiplication factor of light water moderated critical systems.

To test the heavy water library we selected all the heavy water moderated thermal systems available in the ICSBEP Handbook: 8 systems with high enriched uranium dioxide fuel (HEUCOMP-THERM-017), 11 systems with uranyl-fluoride solutions (HEU-SOL-THERM-004 and 020), 10 low enriched uranium dioxide fuel systems (LEU-COMP-THERM-093), one system with natural uranium in metal form (LEU-MET-THERM-001), 34 systems with low enriched uranium in metal form (LEU-MET-THERM-002 and -015) and one system with ${ }^{233} \mathrm{UO}_{2}{ }^{232} \mathrm{ThO}_{2}$ fuel (U233COMP-THERM-004). The systems were calculated using ENDF/B-VII cross sections and the neutron transport code MCNP5-1.60.

When the $\mathrm{D}\left(\mathrm{D}_{2} \mathrm{O}\right)$ thermal scattering library from ENDF/B-VII is replaced with our library for $\mathrm{D}\left(\mathrm{D}_{2} \mathrm{O}\right)$ and $\mathrm{O}\left(\mathrm{D}_{2} \mathrm{O}\right)$, the calculated results (Fig. 3) show an improvement in 39 of the 65 cases (60\%), with differences up to $1100 \mathrm{pcm}$.

\subsection{Conclusions}

In this paper we presented a new set of models for the scattering of thermal neutrons with light and heavy water. In the case of light water, we found an improvement on the calculation of the total cross section below $1 \mathrm{meV}$, but this improvement has little impact on nuclear criticality calculations. On the other hand, the model for heavy water shows an improvement in the calculation of the total cross section below $3 \mathrm{meV}$, but also in the $\sim 20 \mathrm{meV}$ range, which has more importance from the 


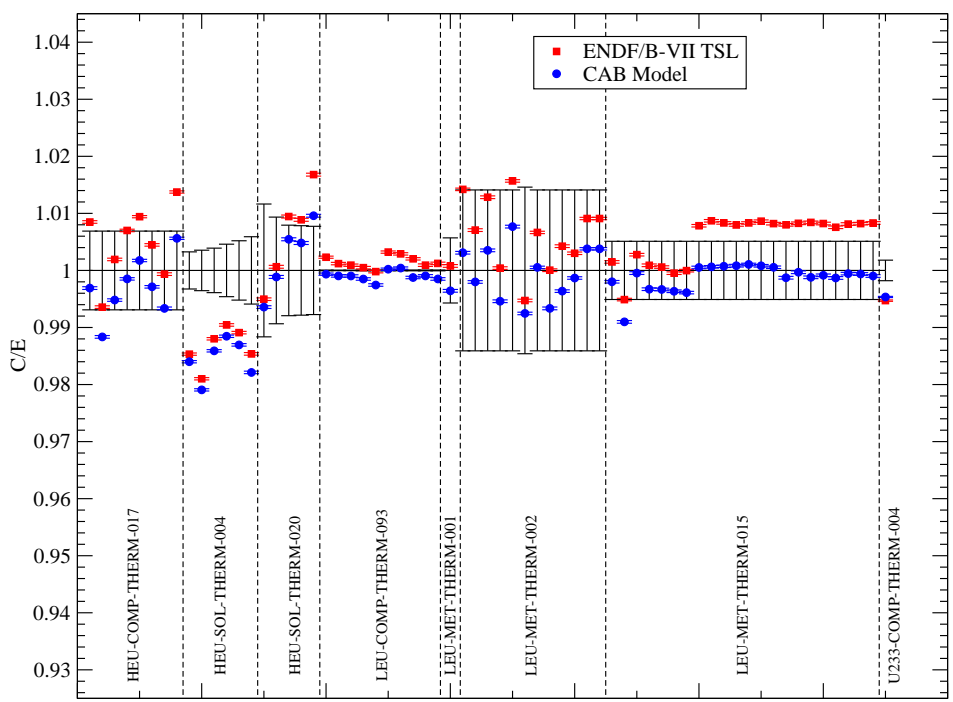

Figure 3: Calculated over experimental (benchmark) ratio for the multiplication factor of heavy water moderated critical systems..

reactor physics perspective. When the heavy water library is applied to the calculation of critical systems, changes of up to $1000 \mathrm{pcm}$ are found.

\section{Acknowledgements}

J.I. Márquez Damián acknowledges the support provided by CONICET. D. C. Malaspina acknowledges the support from NSF (Grant CHE-0957653).

\section{References}

[1] J. Granada, V. Gillette, Evaluation of thermal-neutron cross-sections of moderator materials using a synthetic scattering function, Physica B: Condensed Matter, 213, 821 (1995).

[2] J. Granada, J. Dawidowski, R. Mayer, V. Gilette, Thermal neutron cross section and transport properties of polyethilene, Nucl. Instr. Meth. Phys. Res. A, 261, 573 (1987).

[3] J. Granada, F. Cantargi, J. Marquez Damian, Neutron Cross Sections Libraries for Methane in Phase II and Solid Deuterium, J. Korean Phys. Soc., 59, 1076 (2011).

[4] J. I. Marquez Damian, D. Malaspina, J. Granada, Vibrational spectra of light and heavy water with application to neutron cross section calculations, J. Chem. Phys., 139, 024504 (2013).

[5] J. I. Marquez Damian, D. Malaspina, J. R. Granada, CAB Models for Water: A New Evaluation of the Thermal Neutron Scattering Laws for Light and Heavy Water in ENDF-6 format, Annals of Nuclear Energy, 65, 280 (2014).

[6] A. Trkov, M. Herman, D. E. Brown, ENDF-6 Formats Manual, Tech. Rep. BNL-90365-2009 Rev. 2, Brookhaven National Laboratory, USA (2011). 
[7] J. Koppel, D. Houston, Reference manual for ENDF thermal neutron scattering data, Tech. Rep. GA-8774, ENDF-269, General Atomics (1978).

[8] J. Keinert, M. Mattes, E. Sartori, JEF-1 scattering law data, JEF Report 2/JEF/DOC 41.2, IKE Stuttgart (1984).

[9] K. Shibata, et al., JENDL-4.0: a new library for nuclear science and engineering, J. Nucl. Sci. Technol., 48, 1 (2011).

[10] A. Santamarina, et al., The JEFF-3.1.1 nuclear data library, JEFF Report 22, OECD/NEA (2009).

[11] M. Chadwick, et al., ENDF/B-VII.1 nuclear data for science and technology: Cross sections, covariances, fission product yields and decay data, Nucl. Data Sheets, 112, 2887 (2011).

[12] S. Zabrodskaya, A. Ignatyuk, V. Kosheev, M. Nikolaev, V. Pronyaev, ROSFOND - Russian National Library of Neutron Data, VANT (Voprosi Atomnoy Nauki i Techniki) Ser. Nuclear Constants, 1-2, 3 (2007).

[13] R. MacFarlane, New thermal neutron scattering files for ENDF/B-VI release 2. Tech. Rep. LA-12639-MS, Los Alamos National Laboratory (1994).

[14] M. Mattes, J. Keinert, Thermal neutron scattering data for the moderator materials H2O, D2O and $\mathrm{ZrHx}$ in ENDF-6 format and as ACE library for MCNP (X) codes. Tech. Rep. INDC (NDS)-0470, IAEA (2005).

[15] K. Kozier, D. Roubtsov, R. Rao, J. Svenne, L. Canton, A. Plompen, M. Stanoiu, M. Nankov, C. Rouki, Status of Deuterium Nuclear Data for Simulation of Heavy Water Reactors, in Proceedings of Int. Conf. on Future of Heavy Water Reactors, Ottawa, Ontario, Canada, CNS, 2011, p. 054.

[16] A. Plompen, Summary Report of the Technical Meeting on Long-term Needs for Nuclear Data Development, Tech. Rep. INDC-NDS-0601, IAEA (2012).

[17] D. Van Der Spoel, E. Lindahl, B. Hess, G. Groenhof, A. Mark, H. Berendsen, GROMACS: fast, flexible, and free, J. Comput. Chem., 26, 1701 (2005).

[18] M. González, J. Abascal, A flexible model for water based on TIP4P/2005, J. Chem. Phys., 135, 224516 (2011).

[19] P. Egelstaff, P. Schofield, On the evaluation of the thermal neutron scattering law, Nucl. Sci. and Eng., 12, 260 (1962).

[20] A. Novikov, A. Vankov, L. Gosteva, Temperature dependence of the general spectrum for water, J. Struct. Chem., 31, 77 (1990).

[21] K. Sköld, Small energy transfer scattering of cold neutrons from liquid argon, Phys. Rev. Lett., 19, 1023 (1967).

[22] A. Soper, C. Benmore, Quantum differences between heavy and light water, Phys. Rev. Lett., 101, 065502 (2008).

[23] K. Zaitsev, V. Petrov, S. Kuznetsov, O. Langer, I. Meshkov, A. Perekrestenko, The Total Cross Sections of the Interaction of ultracold Neutrons with H2O and D2O, Atom. Energy, 70, 238 (1991).

[24] J. Briggs, International Handbook of Evaluated Criticality Safety Benchmark Experiments, Tech. Rep. NEA/NSC/DOC(95)3, OECD/NEA (2010).

[25] F. Brown, X-5 Monte Carlo Team, MCNP - A general Monte Carlo N-particle transport code, version 5, Tech. Rep. LA-UR-03-1987, Los Alamos National Laboratory (2003). 